\title{
In vitro antibacterial activity of Bunium persicum and Rheum ribes on Acinetobacter baumanii
}

\author{
Research Article
}

\section{Najme Bagheri ${ }^{1}$, Niloofar Safaei ${ }^{1}$, Elahe Aleebrahim-Dehkordy ${ }^{2}$, Mansoor Khaledi ${ }^{3}$, Mostafa Madmoli ${ }^{4}$, Sadra Ansaripour ${ }^{1^{*}}$}

1. Student Research Committee, Shahrekord University of Medical Sciences, Shahrekord, Iran

2. Young Researchers and Elite Club, Shahrekord Branch, Islamic Azad University, Shahrekord, Iran

3. M.Sc. in Medical Microbiology, Department of Microbiology and Immunology,

Shahrekord University of Medical Sciences, Shahrekord, Iran

4. Emergency Medical Technician, Dezful University of Medical Sciences, Dezful, Iran.

\begin{abstract}
Introduction: Using antibiotics to control pathogenic bacteria is associated with certain side effects in addition to emergence of drug resistance. Nowadays, researchers are considering using plants as suitable alternatives to antibiotics. The aim of study was to compare in vitro antimicrobial activities of aqueous and hydroalcoholic Bunium persicum and Rheum ribes L. extracts on Acinetobacter baumanii. Materials and methods: Different concentrations of R. ribes and B. persicum were prepared to determine microbial sensitivity of A. baumanii using disk diffusion, minimum inhibitory concentration (MIC), and minimum bactericidal concentration (MBC). Results: The highest MIC $(256 \mu \mathrm{g} / \mathrm{ml})$ against A. baumanii was derived for aqueous R. ribes and B. persicum extracts and the lowest MIC $(128 \mu \mathrm{g} / \mathrm{ml})$ for their hydroalcoholic extracts. The highest MBC $(512 \mu \mathrm{g} / \mathrm{ml})$ was derived for aqueous R. ribes extract and the lowest MBC $(256 \mu \mathrm{g} / \mathrm{ml})$ for aqueous and hydroalcoholic R. ribes and B. persicum and hydroalcoholic R. ribes extracts. The extracts exhibited great inhibitory effects against the studied bacteria in disk diffusion; and with increasing the extracts' concentrations, the inhibitory effect was exhibited more markedly as increased diameter of inhibition zone. Conclusion: Hydroalcoholic R. ribes and B. persicum extracts can be used as nature-based compounds to control A. baumanii.
\end{abstract}

Keywords: Plant extract, antimicrobial activity, Acinetobacter baumanii, Bunium persicum, Rheum ribes.

\section{Introduction:}

Acinetobacter baumanii is a gram-negative, nonmotile, and non-fermented coccus that is isolated mainly in hospital settings and ICUs. It is argued that this opportunistic pathogen is associated with nosocomial infections including urinary tract infections (UCIs), surgical site infections, meningitis, and occasionally infections of skin and central nervous system $(1,2)$. This bacterium can grow in a wide range of $\mathrm{pH}$ and environmental temperature and uses different types of substances to grow (3). A. baumanii is widely known mainly because it can survive in both moist and dry environments, and on unsterile medical devices such as ventilators, catheters, and respiratory equipment (4). Due to wide and frequent therapeutic treatments, this pathogen has acquired resistance to drugs, even strong ones, across the world. Although pharmaceutical and pharmacological industries have produced some new antibiotics during the last three decades,

\section{*Corresponding Author:}

\section{Sadra Ansaripour,}

Student Research Committee,

Shahrekord University of Medical Sciences,

Shahrekord, Iran.

E-mail: ansaripour_sadra@yahoo.com microorganisms' resistance to these antibiotics has increased. Overall, it is obvious that bacteria can genetically be converted and resist therapeutic agents. In addition to increasing healthcare costs, this multidrug resistance has increased mortality rate and its spread to the environments outside hospital settings (5). This issue should be seriously and even worriedly taken into consideration, especially for immunosuppressive patients who are faced with certain bacteria in hospitals that acquire double and even greater resistance, and therefore development of new infections can lead to higher rates of mortality (6). The problem with such microbial resistance is uncertainty about antimicrobial drugs in the future. Therefore, certain measures should be designed to decrease this problem including controlling use of antibiotics, developing research to better understand the genetic mechanism of this resistance, and conducting research to develop new drugs (7). Currently, more potent therapeutic agents should be sought out and used to fight antibiotic resistant bacteria particularly extensive drug resistant (XDR) ones including A. baumanii. Using medicinal plants is a strategy to achieve this purpose. Certain medicinal plants have been used to develop such therapeutic methods, due to bioactive compounds present in them, to investigate their potential use. For 
thousands of years, plant extracts have been used for various purposes. Particularly, many applications such as preservatives, pharmaceutical items, alternative medicine, and nature-based therapies have been based on the antimicrobial activities of plant essences and extracts. Therefore, it would be a promising strategy to discover medicinal plants with antimicrobial effects that are economical due to causing comparatively fewer side effects and exerting less toxicity on tissues (8).

Rhubarb, botanically referred to as Rheum Ribes L., is from family Polygonaceae. Different species of this plant occur in different regions of the world including China, India, Turkey, and Iran. R. ribes is a type of resistant perennial plant that is cultivated in some countries due to its edible red part (9). R. ribes occurs in different regions of Iran especially Mashhad and Nishabur. In traditional medicine books, several properties have been mentioned for $\mathrm{R}$. ribes including appetizing, blood-purifying, bile reducing, gastrointestinal tract especially liver-enhancing, bile and phlegm-excreting, hepatoprotective, and antidotic for certain toxins (10). This plant has been frequently studied and reported to have various properties including antiplatelet accumulation, hypolipidemic, kidney failure and disease-treating, pancreatic tissue-improving in diabetic people, and fat accumulation-reducing in the liver (11).

Black cumin (Bunium persicum) is an aromatic perennial plant from family Umbelliferae that occurs from Central Asia to northern India. Its seeds are widely used as spice. In traditional medicine, it is used as antiflatulence agent as well as in treating indigestion (12). B. persicum has a great potential for pharmaceutical applications due to antispasmodic, cancer-preventive, cell proliferation-controlling, acid secretion-reducing, mucin secretion-increasing, prostaglandin 2-increasing, and leukotriene-reducing (13). B. persicum wildly occurs in the regions of Iran with dry climate such as Kerman, Fars, Isfahan, and Yazd provinces (14).

With regards to the significance of A. baumanii in spreading diseases and importance of using phytotherapy as well as because no study has yet been conducted to investigate in vitro antibacterial effects of $\mathrm{R}$. ribes and $\mathrm{B}$. persicum, this study was conducted to investigate the antibacterial effects of hydroalcoholic and aqueous R. ribes and B. persicum aerial parts extracts on A. baumanii.

\section{Materials and methods Extraction}

To prepare hydroalcoholic extract, $500 \mathrm{~g}$ of the studied plants were pulverized with a mill and extracted by maceration. To do this, $500 \mathrm{~g}$ of each plant was kept in combination with alcohol $70 \%$ in 1-liter volume for four days. After being filtered with Whatman filter paper, the solution was concentrated in rotary at $40{ }^{\circ} \mathrm{C}$. After distillation, the solution was incubated at $37^{\circ} \mathrm{C}$ and the extract obtained. Then, a stock solution was prepared from the extract using dimethyl sulfoxide.

\section{Preparation of microbial suspension}

Twenty four hours before the study, a fresh (24hour) culture medium was prepared by means of already prepared culture media such that before onset of inoculation, an amount of the colonies on the surface of Mueller-Hinton broth was transferred into a physiological serum-containing tube with a sterile swab and then microbial suspension opacity prepared and compared with 0.5 McFarland standard opacity.

\section{Bacteria and antibiotics}

Standard A. Baumanii strain (ATCC747), purchased from Iranian Research Organization for Science and Technology as lyophilized, was used in this study. The antibiotics used in this study were amikacin, imipenem, ceftazidime, gentamicin, and ciprofloxacin (Padtan Teb Co., Iran).

\section{Tests of determining drug sensitivity (antibiogram)}

To conduct antibiogram, disk diffusion was used. For this purpose, paper sterile blank disks (Padtan Teb Co., Iran) were placed in prepared concentrations of the extract for 24 hours so that the extract was completely absorbed by the disks. Then, the disks containing the extracts in various concentrations were incubated at $37{ }^{\circ} \mathrm{C}$ for one hour to dry and then cultured on the plates containing bacteria alongside the standard disks containing amikacin, imipenem, ceftazidime, gentamicin, and ciprofloxacin at appropriate distances and incubated at $37{ }^{\circ} \mathrm{C}$ for one hour to examine the extract for antibacterial activity. Finally, inhibition zone diameters were measured by ruler. To achieve this purpose, the ruler's zero edge was placed next to the diameter of the inhibition zone to measure the distance to where the inhibition zone end was placed on the ruler. The inhibition zone diameters were reported in $\mathrm{mm}$ and the results were compared with CLSI2013.

\section{Determining minimum inhibitory concentration (MIC)}

MICs were determined according to the CLSI protocol (2013). First, a stock solution was prepared from the extract and then the extract was diluted in ELISA wells containing TSB culture medium in serial manner (1/2 dilution). After preparing bacterial suspension according to $0.5 \mathrm{McF}$ arland Standard, 100 $\mu \mathrm{l}$ of the bacterial suspension was added to each well and incubation was performed at $37^{\circ} \mathrm{C}$ for one hour. After bacterial growth, the MICs were determined.

\section{Determining minimum bactericidal concentration (MBC)}

After determining the MICs, $20 \mu \mathrm{l}$ of the solutions of the tubes whereby the bacteria did not grow was removed and cultured on blood agar medium. After 24-hour incubation at $37{ }^{\circ} \mathrm{C}$, the least concentration of the extract in which the bacteria was unable to grow was considered MBC. 
Statistical analysis

The diameters of all inhibition zones for R. ribes, B. persicum, and positive control were measured and analyzed by comparing mean values. All tests were conducted in triplicate.

\section{Results}

In this study, the antimicrobial activities of aqueous and hydroalcoholic R. ribes and B. persicum extracts were measured on A. baumanii; and the inhibitory effects of these extracts were measured with reference to presence or absence of inhibition zone, and the MICs and MBCs were measured.

The results on hydroalcoholic R. ribes and B. persicum extracts indicated that the MIC and MBC of both extracts were 128 and $256 \mu \mathrm{g} / \mathrm{ml}$, respectively, for A. baumanii. In addition, the results on aqueous R. ribes and B. persicum extracts showed that the MIC of both extracts was $256 \mu \mathrm{g} / \mathrm{ml}$, and the MBC of R. ribes extract was higher $(512 \mu \mathrm{g} / \mathrm{ml})($ Table 1$)$.

Table 1. Effect of hydroalcoholic and aqueous extracts of the plants investigated on Acinetobacter baumanii

\begin{tabular}{|l|c|c|c|c|}
\hline \multirow{2}{*}{ Plant } & \multicolumn{2}{|c|}{ Hydroaclcoholic extract } & \multicolumn{2}{c|}{ Aqueous extract } \\
\cline { 2 - 5 } & $\mathrm{MIC}(\mu \mathrm{g} / \mathrm{ml})$ & $\mathrm{MBC}(\mu \mathrm{g} / \mathrm{ml})$ & $\mathrm{MIC}(\mu \mathrm{g} / \mathrm{ml})$ & $\mathrm{MBC}(\mu \mathrm{g} / \mathrm{ml})$ \\
\hline Bunium persicum & 128 & 256 & 256 & 256 \\
\hline Rheum ribes & 128 & 256 & 256 & 512 \\
\hline
\end{tabular}

The highest MIC $(256 \mu \mathrm{g} / \mathrm{ml})$ against A. baumanii was derived for aqueous B. persicum and R. ribes extracts and the lowest MIC $(128 \mu \mathrm{g} / \mathrm{ml})$ for their hydroalcoholic extracts. The highest MBC $(512 \mu \mathrm{g} / \mathrm{ml}) \mathrm{was}$ derived for aqueous $\mathrm{R}$. ribes extract and the lowest $\mathrm{MBC}(256 \mu \mathrm{g} / \mathrm{ml})$ for aqueous and hydroalcoholic B. persicum and hydroalcoholic R. ribes extracts. The MIC and MBC of hydroalcoholic B. persicum extract were lower than those of aqueous $\mathrm{R}$. ribes extract.

In disk diffusion, the inhibition zone diameters in different groups (Tables 2 and 3) indicated that the greatest effect was exerted by hydroalcoholic B. persicum extract $(28 \mathrm{~mm})$ and the least effect by aqueous R. ribes extract in $512 \mu \mathrm{g} / \mathrm{ml}(7 \mathrm{~mm})$. Inhibition zone diameter was directly correlated with the extracts' antibacterial activities. No inhibition zone was seen surrounding the blank disk that was considered to represent negative control.

Table 2. Zone diameters of growth inhibition (in $\mathrm{mm}$ ) caused by aqueous Bunium persicum and $\mathrm{Rheum}$ ribes extract at different concentrations with disk diffusion method

\begin{tabular}{|c|c|c|c|c|c|c|c|}
\hline $\begin{array}{c}\text { Different concentrations } \\
\text { Extract }\end{array}$ & $\begin{array}{c}\mathbf{8}(\mu \mathrm{g} / \\
\mathbf{m l})\end{array}$ & $\begin{array}{c}16(\mu \mathrm{g} / \\
\mathbf{m l})\end{array}$ & $\begin{array}{c}32(\mu \mathrm{g} / \\
\mathbf{m l})\end{array}$ & $\begin{array}{c}\mathbf{6 4}(\boldsymbol{\mu g} / \\
\mathrm{ml})\end{array}$ & $\begin{array}{c}128(\mu \mathrm{g} / \\
\mathrm{ml})\end{array}$ & $\begin{array}{c}256(\mu \mathrm{g} / \\
\mathrm{ml})\end{array}$ & $\begin{array}{c}512(\mu \mathrm{g} / \\
\mathrm{ml})\end{array}$ \\
\hline Bunium persicum & - & - & - & - & - & $23 \mathrm{~mm}$ & $27 \mathrm{~mm}$ \\
\hline Rheum ribes & - & - & - & - & $2 \mathrm{~mm}$ & $4 \mathrm{~mm}$ & $7 \mathrm{~mm}$ \\
\hline
\end{tabular}

*-Demonstrate bacterium resistance

Gentamicin:15mm, Imipenem:16mm, Amikacin:17mm, Ceftazidime:18mm, Ciprofloxacin:21mm

Table 3. Zone diameters of growth inhibition (in $\mathrm{mm}$ ) caused by hydroalcoholic Bunium persicum and $\mathrm{Rheum}$ ribes extract at different concentrations with disk diffusion method

\begin{tabular}{|c|c|c|c|c|c|c|c|}
\hline $\begin{array}{c}\text { Different concentrations } \\
\text { Extract }\end{array}$ & $\begin{array}{c}8(\mu \mathrm{g} / \\
\mathrm{ml})\end{array}$ & $\begin{array}{c}16(\mu \mathrm{g} / \\
\mathrm{ml})\end{array}$ & $\begin{array}{c}32(\mu \mathrm{g} / \\
\mathrm{ml})\end{array}$ & $\begin{array}{c}64(\mu \mathrm{g} / \\
\mathrm{ml})\end{array}$ & $\begin{array}{c}128(\mu \mathrm{g} / \\
\mathrm{ml})\end{array}$ & $\begin{array}{c}256(\mu \mathrm{g} / \\
\mathrm{ml})\end{array}$ & $\begin{array}{c}512(\mu \mathrm{g} / \\
\mathrm{ml})\end{array}$ \\
\hline Bunium persicum & - & - & - & - & $15 \mathrm{~mm}$ & $20 \mathrm{~mm}$ & $28 \mathrm{~mm}$ \\
\hline Rheum ribes & - & - & $2 \mathrm{~mm}$ & $4 \mathrm{~mm}$ & $8 \mathrm{~mm}$ & $10 \mathrm{~mm}$ & $12 \mathrm{~mm}$ \\
\hline
\end{tabular}

*-Demonstrate bacterium resistance

Gentamicin:15mm, Imipenem:16mm, Amikacin:17mm, Ceftazidime:18mm, Ciprofloxacin:21mm 


\section{Discussion}

Experimental evidence and using plants as drugs offer basic information on usefulness of plant extracts in special medical conditions. Since the past, plant extracts such as those of tea tree and clove tree have been used for topical treatment due to antimicrobial properties $(15,16)$. It is essential to examine the antimicrobial properties of such plants that have already been used in traditional medicine (17). In addition, using the compounds that are welcome by consumers, naturebased, and safe further highlights the need for supplementing information on these plant-based extracts and compounds. Studies have confirmed the activities of the essences and extracts derived from various plants such as rosemary, peppermint, basil, tea tree, celery seeds, and fennel $(8,18)$.

The current study demonstrated that hydroalcoholic R. ribes and aqueous and hydroalcoholic B. persicum extracts exhibited adequate antibacterial activity against $\mathrm{A}$. baumanii. The greatest MIC against A. baumanii was derived for aqueous B. persicum and R. ribes extracts and the lowest MIC for their hydroalcoholic extracts. The highest MBC was derived for aqueous $\mathrm{R}$. ribes extract and the lowest $\mathrm{MBC}$ for aqueous and hydroalcoholic B. persicum and hydroalcoholic R. ribes extracts.

It has been reported that generally, phenolic compounds are active compounds of plant extracts against A. baumanii, a gram-negative bacterium (19). Many compounds isolated from plants whose activities have been demonstrated are secondary compounds that have been isolated using solvents such as methanol, ethanol, water, and acetone as well as by different techniques. A study on antibacterial activity of alcoholic R. ribes extract against microorganisms from genuses Escherichia coli, Pseudomonas, Shigella, Bacillus, Staphylococcus, and Micrococcus, demonstrated that R. ribes exhibited antimicrobial activity (20). Antimicrobial agents are certain polyphenols such as simple phenols, phenolic acids, quinones, flavones, flavonoids, flavonols, tannins, and coumarines. Besides that, terpenoids, essential oils, alkaloids, lactines, polypeptides, etc. have been reported. The action mechanisms of these compounds depends on their types. These mechanisms include inhibiting enzymatic activity, plant extract active compounds' combining with extracellular proteins, and solving microbe's or compound's cell with bacterial cell wall (21). Certain extracts can disrupt microorganism DNA, which may occur as development of ion channels in the microbial membrane or competing with the microbial proteins for binding to the host's polysaccharide receptors (22).

A study on cinnamon's activity against drug resistant A. baumanii demonstrated that cinnamon's aldehydes prevented the decarboxylating activities of amino acids in bacteria but could not degenerate extracellular membrane or evacuate intracellular ATP (21). Hydroxyl groups of phenolic compounds can degenerate bacterial cell wall membrane. More clearly, these groups can decrease the amount of intracellular ATP and membrane potential (23). In addition, the secretion of certain substances such as ATP, amino acids, ions, and nucleic acids can ultimately lead to bacterial death. Turmeric has been reported to exert antibacterial activity against Acinetobacter for certain reasons such as disrupting folic acid metabolism and cell wall gap (24).

$\mathrm{R}$. ribes has already been demonstrated to exert significant antimicrobial effects on bacterial genuses such as Pseudomonas, Klebsiella, E. coli, etc. (9). Cumin exhibited potent inhibitory effect against Listeria monocytogenes. It has been argued that the terpens in cumin essence caused inflammation of cytoplasmic membrane and disruption of microorganism membrane (12).

Karaman et al. studied antimicrobial activity of alcoholic Juniperus oxycedrus leaf extract on a wide spectrum of bacteria including gram-negative, grampositive, and fungal bacteria. Their results demonstrated that aqueous Juniperus oxycedrus extract had no antimicrobial activity against bacterial and fungal microorganisms. The highest $\mathrm{MIC}$ and $\mathrm{MBC}$ of alcoholic extract of this plant ranged $7-17 \mathrm{~mm}$ for sensitive microorganisms (25). In addition, evidence has indicated that ethanol is $\mathrm{s}$ better solvent to isolate the antimicrobial compounds from medicinal plants compared to other solvents such as water and hexane (26). Differences in the sensitivity rates of microorganisms against antimicrobes in plant extracts can be explained by different cell wall compounds or the specific genetics of the species in plasmid that can easily vary among different bacterial species (25). It is relatively difficult to compare the current study's findings with other's, which can be due to different compounds in different plant-based essences and extracts possibly because of native climatic and ecological conditions of the plants. In addition, it is likely that some compounds with conventional similar names are isolated from different plants, and the methods of assessing antimicrobial activity and selected tests different among findings (21).

Briefly, this study confirmed that these extracts, with antibacterial properties and if further studied, can be used as antibacterial agents for treating infectious diseases. As well, with regards to the effects of these extracts on life and proliferation of $\mathrm{A}$. baumanii, they can be used as drugs to prevent the wounds due to this bacterium.

\section{Conclusion}

This study was first to show moderate to high antibacterial effects of hydroalcoholic and aqueous $\mathrm{R}$. ribes and $\mathrm{B}$. persicum extracts that varied with changing the extracts' concentrations. Aqueous extracts exerted the greatest effect and can therefore be used as a potent 
antimicrobial agent, drug supplement, and alternative treatment. Moreover, it is essential to conduct further experimental studies and clinical trials to investigate inhibitory effects of these extracts on A. baumanii.

\section{Acknowledgements}

This study was derived from a research project approved at the Students Research Committee of the Shahrekord University of Medical Sciences (no.: 2552). The authors acknowledge the funding of the Research and Technology Deputy and cooperation of the Medical Plants Research Center of this university.

\section{Conflict of interest}

There are no conflicts of interest in this study.

\section{References}

1. Zarrilli R, Giannouli M, Tomasone F, Triassi M, Tsakris A. Carbapenem resistance in Acinetobacter baumannii: the molecular epidemic features of an emerging problem in health care facilities. J. Infect. Dev. Countries. 2009;3(05):335-41.

2. Zhang H-z, Zhang J-s, Qiao L. The Acinetobacter baumannii group: a systemic review. World J. Emerg. Med. 2013;4(3):169.

3. Tiwari V, Tiwari M. Quantitative proteomics to study carbapenem resistance in Acinetobacter baumannii. Front. Microbiol. 2014;5:512.

4. Tiwari V, Tiwari M. Phosphoproteomics as an emerging weapon to develop new antibiotics against carbapenem resistant strain of Acinetobacter baumannii. J. Proteomics. 2015; 112:336-8.

5. Peleg AY, Seifert H, Paterson DL. Acinetobacter baumannii: emergence of a successful pathogen. Clin. Microbiol. Rev. 2008;21(3):538-82.

6. Cohen ML. Epidemiology of drug resistance: implications for a post-antimicrobial era. Science. 1992;257(5073):1050-5.

7. Nascimento S, Chiappeta AdA, Lima R. Antimicrobial and cytotoxic activities in plants from Pernambuco, Brazil. Fitoterapia. 1990;61(4):353-5.

8. Lis $\square$ Balchin M, Deans S. Bioactivity of selected plant essential oils against Listeria monocytogenes. J. Appl. Microbiol. 1997;82(6):759-62.

9. Bazzaz F, Khajehkaramadin M, Shokooheizadeh HR. In vitro antibacterial activity of Rheum ribes extract obtained from various plant parts against clinical isolates of Gram-negative pathogens. Iranian $\mathrm{J}$. Pharm. Res. 2010:87-91.

10. Li LS, Liu ZH. Clinical and experimental studies of rheum on preventing progression of chronic renal failure. Zhongguo Zhong $\mathrm{Xi}$ Yi Jie $\mathrm{He} \mathrm{Za}$ Zhi. 1991;11(7):392-6.

11. Hamzeh S, Farokhi F, Heidari R, Manaffar R. The Effect of Hydroalcoholic Extract of Rhubarb Root on Blood Glucose and Histopathological Changes of
Pancreas in Alloxan-induced Diabetic Rats. Qom Univ. Med. Sci. 2015;8(5).

12. Rabiey S, Hosseini H, Rezaei M. The hurdle effect of Bunium persicum essential oil, smoke and $\mathrm{NaCl}$ for controlling the Listeria monocytogenes growth in fish model systems. J. Food Saf. 2013;33(2):137-44.

13. Eddouks M, Lemhadri A, Michel J-B. Caraway and caper: potential anti-hyperglycaemic plants in diabetic rats. J. Ethnopharmacol. 2004;94(1):143-8.

14. De Carvalho CC, da Fonseca MMR. Carvone: Why and how should one bother to produce this terpene. Food Chem. 2006;95(3):413-22.

15. Hoffman D. The herb user's guide. Wellingborough, UK: Thorsons Publishing Group. 1987.

16. Aleebrahim-Dehkordy E, Ansaripour S, RafieianKopaei M, Saberianpour S. Effects of substances on plants' active compounds on changes in the hormone levels of the pituitary-thyroid axis in hyperthyroidism and hypothyroidism. Pharmacogn. Rev. 2018;12(23): 1 .

17. Mitscher LA, Drake S, Gollapudi SR, Okwute SK. A modern look at folkloric use of anti-infective agents. J. Nat. Prod. 1987;50(6):1025-40.

18. Yousef R, Tawil G. Antimicrobial activity of volatile oils. Die Pharmazie. 1979;35(11):698-701.

19. Miyasaki Y, Rabenstein JD, Rhea J, Crouch M-L, Mocek UM, Kittell PE, et al. Isolation and characterization of antimicrobial compounds in plant extracts against multidrug-resistant Acinetobacter baumannii. PloS one. 2013;8(4):e61594.

20. Eliopoulos GM, Maragakis LL, Perl TM. Acinetobacter baumannii: epidemiology, antimicrobial resistance, and treatment options. Clin. Infect. Dis. 2008;46(8):1254-63.

21. Burt S. Essential oils: their antibacterial properties and potential applications in foods - a review. Int. J. Food Microbiol. 2004;94(3):223-53.

22. Hili P, Evans C, Veness R. Antimicrobial action of essential oils: the effect of dimethylsulphoxide on the activity of cinnamon oil. Lett. Appl. Microbiol. 1997;24(4):269-75.

23. Ultee A, Bennik M, Moezelaar R. The phenolic hydroxyl group of carvacrol is essential for action against the food-borne pathogen Bacillus cereus. Appl. Environ. Microbiol. 2002;68(4):1561-8.

24. Rai D, Singh JK, Roy N, Panda D. Curcumin inhibits FtsZ assembly: an attractive mechanism for its antibacterial activity. Biochem. J. 2008;410 (1):147-55.

25. Karaman I, Şahin F, Güllüce $M$, Ögüüçü $H$, Şengül M, Adigüzel A. Antimicrobial activity of aqueous and methanol extracts of Juniperus oxycedrus L. J. Ethnopharmacol. 2003;85(2):231-5.

26. Eloff J. Which extractant should be used for the screening and isolation of antimicrobial components from plants? J. Ethnopharmacol. 1998;60(1):1-8. 